\title{
Alterações na eficiência do uso da água relacionadas com fatores climáticos e uso e ocupação do solo, na região do MATOPIBA
}

\author{
Changes in water use efficiency related to climatic factors and land use and occupation in the \\ MATOPIBA region \\ Cambios en la eficiencia del uso del agua relacionados con factores climáticos y uso y ocupación del \\ suelo en la región de MATOPIBA
}

Recebido: 05/07/2021 | Revisado: 08/07/2021 | Aceito: 08/07/2021 | Publicado: 19/07/2021

\author{
Dimas de Barros Santiago \\ ORCID: https://orcid.org/0000-0001-7118-8467 \\ Universidade Federal de Campina Grande, Brasil \\ E-mail: dimas.barros91@gmail.com \\ Humberto Alves Barbosa \\ ORCID: https://orcid.org/0000-0002-9641-806X \\ Universidade Federal de Alagoas, Brasil \\ E-mail: barbosa33@gmail.com \\ Washington Luiz Félix Correia Filho \\ ORCID: https://orcid.org/0000-0002-4029-4491 \\ Universidade Federal de Alagoas, Brasil \\ E-mail: wlfcfm@gmail.com
}

\begin{abstract}
Resumo
O estudo tem por objetivos: estimar e analisar as mudanças espaço-temporais da Eficiência do Uso da Água (Water Use Efficiency - WUE) no MATOPIBA e avaliar a influência de fatores climáticos e do uso e ocupação do solo na variação do WUE. No estudo serão utilizados os produtos MOD17A2 (GPP) e MOD16A2 (ET) derivados do sensor MODIS (Moderate Resolution Imaging Spectroradiometer) obtidos no United States Geological Survey (USGS), com resolução espacial $1 \mathrm{~km}$ x 1km, para as computo da WUE anual no período entre $2001 \mathrm{e} 2019$. Em relação a avaliação do uso da terra será realizado com as imagens do MAPBIOMAS, com resolução de $30 \mathrm{~m}$ x 30m. Já os dados de precipitação serão provenientes do Climate Hazard Group InfraRed Precipitation with Station data (CHIRPS) com resolução espacial de $5,6 \mathrm{~km}$ por $5,6 \mathrm{~km}$. As realizações dos cálculos matemáticos serão executadas nos softwares ambiente R versão 3.6-3 e Quantum GIS versão 3.4-6. Os resultados apontam que os maiores (menores) valores de WUE ocorrem em regiões agrícolas e áreas de vegetação nativa amazônica (áreas de superfícies não vegetadas). Este padrão da WUE está associado à crescente expansão agrícola sobre as regiões (Oeste Baiano e na porção Piauí), motivados principalmente pelo plantio de soja. Além disso, constatou-se que as anomalias positivas (negativas) da WUE ocorrem em anos secos (chuvosos). Concluindo assim que, áreas agrícolas são propensas aos maiores valores da WUE devido ao manejo cultural auxiliando no desenvolvimento das culturas agrícolas. As respostas da vegetação aos eventos secos e chuvosos foram mais sensíveis em áreas agrícolas que em áreas de vegetação nativa.

Palavras-chave: Eficiência do uso da água; MATOPIBA; Uso e ocupação do solo.
\end{abstract}

\begin{abstract}
The study aims to: estimate and analyze the spatial-temporal changes of the WUE in MATOPIBA and evaluate the influence of climatic factors and land use and occupation on the variation of the WUE. The study will use the products MOD17A2 (GPP), and MOD16A2 (ET) derived from the MODIS sensor (Moderate Resolution Imaging Spectroradiometer) obtained in the United States Geological Survey (USGS), with spatial resolution $1 \mathrm{~km} \mathrm{x} 1 \mathrm{~km}$, for the computation of the annual WUE in the period between 2001 and 2019. Regarding the evaluation of land use based on the MAPBIOMAS maps, with a resolution of $30 \mathrm{~m}$ x 30m. Precipitation data will come from the Climate Hazard Group InfraRed Precipitation with Station data (CHIRPS) with a spatial resolution of $5.6 \mathrm{~km}$ by $5.6 \mathrm{~km}$. The mathematical calculations were developed in R environment software version 3.6-3 and Quantum GIS version 3.4-6. The results show that the highest (lowest) values of WUE occur in agricultural regions and areas of native Amazonian vegetation (non-vegetated surface areas). This WUE pattern is associated with the growing agricultural expansion over the regions (West Bahia and in the Piauí portion), mainly motivated by soy planting. In addition, during dry (wet) years have positive (negative) anomalies of WUE. Concluding that, agricultural areas are prone to higher WUE values due to cultural management helping develop crops. Vegetation responses to dry and rainy events were more sensitive in agricultural areas than in areas of native vegetation.
\end{abstract}

Keywords: Water use efficiency; MATOPIBA; Land use and occupation. 


\section{Resumen}

El estudio tiene como objetivo: estimar y analizar los cambios espacio-temporales de la WUE en MATOPIBA y evaluar la influencia de los factores climáticos y el uso y ocupación del suelo en la variación de la WUE. El estudio utilizará los productos MOD17A2 (GPP) y MOD16A2 (ET) derivados del sensor MODIS (espectrorradiómetro de imágenes de resolución moderada) obtenido en el Servicio Geológico de Estados Unidos (USGS), con resolución espacial de $1 \mathrm{~km}$ x $1 \mathrm{~km}$, para el cálculo de la WUE anual en el período comprendido entre 2001 y 2019. En cuanto a la evaluación del uso del suelo, se realizará con las imágenes de MAPBIOMAS, con una resolución de 30m x 30m. Los datos de precipitación provendrán de los datos de precipitación infrarroja con estación (CHIRPS) del Climate Hazard Group con una resolución espacial de $5,6 \mathrm{~km}$ por $5,6 \mathrm{~km}$. Los cálculos matemáticos se realizarán en el software de entorno R versión 3.6-3 y Quantum GIS versión 3.4-6. Los resultados muestran que los valores más altos (más bajos) de WUE ocurren en regiones agrícolas y áreas de vegetación amazónica nativa (áreas de superficie sin vegetación). Este patrón de WUE está asociado con la creciente expansión agrícola en las regiones (West de la Bahia y en la porción de Piauí), principalmente motivada por la siembra de soja. Además, se encontraron anomalías positivas (negativas) de WUE en años secos (húmedos). Concluyendo que, las áreas agrícolas son propensas a valores de WUE más altos debido a que la gestión cultural ayuda al desarrollo de cultivos agrícolas. Las respuestas de la vegetación a eventos secos y lluviosos fueron más sensibles en áreas agrícolas que en áreas de vegetación nativa.

Palabras clave: Eficiencia en el uso del agua; MATOPIBA; Uso y ocupación de la tierra.

\section{Introdução}

Nos últimos anos, o consórcio agrícola composto pelos estados Maranhão, Tocantins, Piauí e Bahia (MATOPIBA) tem se destacado por sua crescente produção de milho, feijão, algodão (Landau et al., 2014), e principalmente na grande produção de soja (Sá et al., 2015), isso devido ao seu desenvolvimento rápido ter sido impulsionado pelos investimentos nas atividades agrícolas (Colussi, 2017), aumentando a economia local.

O aumento populacional no decorrer nas últimas décadas tende ao aumento da demanda por alimentos e água em outros setores (Coelho et al., 2005; Correia Filho et al., 2019; Nicodemo et al., 2021), com isso, áreas de vegetação nativa têm sido convertida em áreas agrícolas.

No setor agrícola, tem sido um grande desafio produzir mais alimentos com menos água, uma meta que só poderia ser alcançada aumentando a produtividade das plantações (Kijne et al., 2003; Zwart \& Bastiaanssen, 2003). Já que as atividades agrícolas são responsáveis pela maior porcentagem (entre 80-90\%) de utilização da água pelo ser humano (Morison et al., 2008).

Estimar o consumo hídrico pelas culturas agrícolas é um dos principais parâmetros para o planejamento correto, auxiliando no dimensionamento e manejo de qualquer sistema de irrigação, bem como para a gestão dos recursos hídricos (Bernardo et al., 2005), obtendo altas produtividades de forma eficiente.

A eficiência do uso da água (WUE) utiliza a relação entre a produtividade primária bruta (PPB) da cultura ou vegetação e a evapotranspiração (ET), já que as trocas de carbono e água entre a vegetação e a atmosfera (fotossíntese), desempenham papel importante no ecossistema (Dalastra et al., 2020; Yamori, 2020). Além de ser uma informação importante utilizada para a avaliação dos impactos referentes as mudanças climáticas, deficiência da irrigação e gestão das estratégias afins de produtividades do ecossistema (Tang et al., 2015a; Tang et al., 2015b).

As alterações em áreas naturais e agrícolas, seja de forma humana ou climática, causam modificações na evapotranspiração e na absorção de carbono, influenciando na eficiência do uso da água. A WUE pode ser alterados em diferentes escalas temporais e espaciais por alguns efeitos, como a área foliar, o tipo de vegetação, a quantidade de radiação absorvida, a precipitação e a radiação (Liu et al., 2015).

As informações sobre a forma como a vegetação (agrícola e nativa) realizam o ciclo carbono-água e fatores que as modifica pode fornecem implicações sobre respostas às mudanças climáticas e ambientais, dando assim, meios para estudos sobre formas de mitigar as consequências.

A WUE vem sendo associados ao clima (Mbava et al., 2020), onde foi destacado a sensibilidade das culturas aos efeitos do padrão de precipitação, tipo de solo e regime climático. WUE e culturas agrícolas (Xiao et al., 2013), mostrando que 
o aquecimento do clima e à queda nas chuvas nos últimos 50 anos, aumentou significativamente a eficiência do uso da água pelo trigo (Triticum aestivum), batata (Solanum tuberosum) e milho (Zea mays), o que mostra que as mudanças climáticas podem melhorar a eficiência do uso da água.

Com isso, a informação proposta pela razão entre a absorção de carbono (GPP) e a perda de água pelas culturas (ET) de forma remota (sensoriamento remoto), traz um gama de informações valiosas não apenas como as culturas respondem as modificações do clima, bem como as modificações antropogênicas.

Esses estudos de forma remota (sensoriamento remoto), facilitam a avaliação em larga escala espacial. Pois em escalas pontuais, através de observações, estações e simulações, não satisfazem as necessidades de informações para uma pesquisa de grande escala. Portanto, o monitoramento via sensoriamento remoto é cada vez mais utilizado, possibilitando informações sobre os processos na superfície. Com isso uma análise sobre como o processo de expansão agrícola impactam na dinâmica dos recursos hídricos no MATOPIBA e nas regiões próximas, através do indicador da eficiência do uso da água (WUE), bem como as consequências das formas de uso e ocupação das terras, trará informações promissoras auxiliando em tomadas de decisões acerca da questão hídrica, principalmente em áreas de produção agrícola.

Desta forma os objetivos são: 1) estimar e analisar as mudanças espaço-temporais da WUE no MATOPIBA e 2) avaliar a influência de fatores climáticos e do uso e ocupação do solo na variação da WUE.

\section{Material e Métodos}

\section{1 Área de Estudo}

A região do MATOPIBA abrange 4 estados brasileiros: MAranhão, TOcantins, PIauí e BAhia, ao qual estima-se que possui 73 milhões de hectares (ha), constituída por 31 microrregiões e 337 municípios (Miranda, Magalhães \& Carvalho, 2014), ilustrada na Figura 1. Em relação à altitude, varia entre 1 e 1.254 metros acima do nível do mar, com áreas elevadas na região do extremo oeste baiano e as menores ao norte maranhense. O MATOPIBA é constituído de 3 biomas: Cerrado (90,94\%), Amazônia $(7,27 \%)$ e Caatinga (1,64\%), com uma área de 66.543.540,87 ha $\left(665.435,41 \mathrm{~km}^{2}\right), 5.319 .628,40$ ha $\left(53.196,29 \mathrm{~km}^{2}\right)$ e 1.203.107,22 ha $\left(12.031,08 \mathrm{~km}^{2}\right)$, respectivamente (Miranda, 2015).

Figura 1 - Localização geográfica da região do MATOPIBA: (a) no Brasil e América do Sul, e (b) elevação do MATOPIBA.
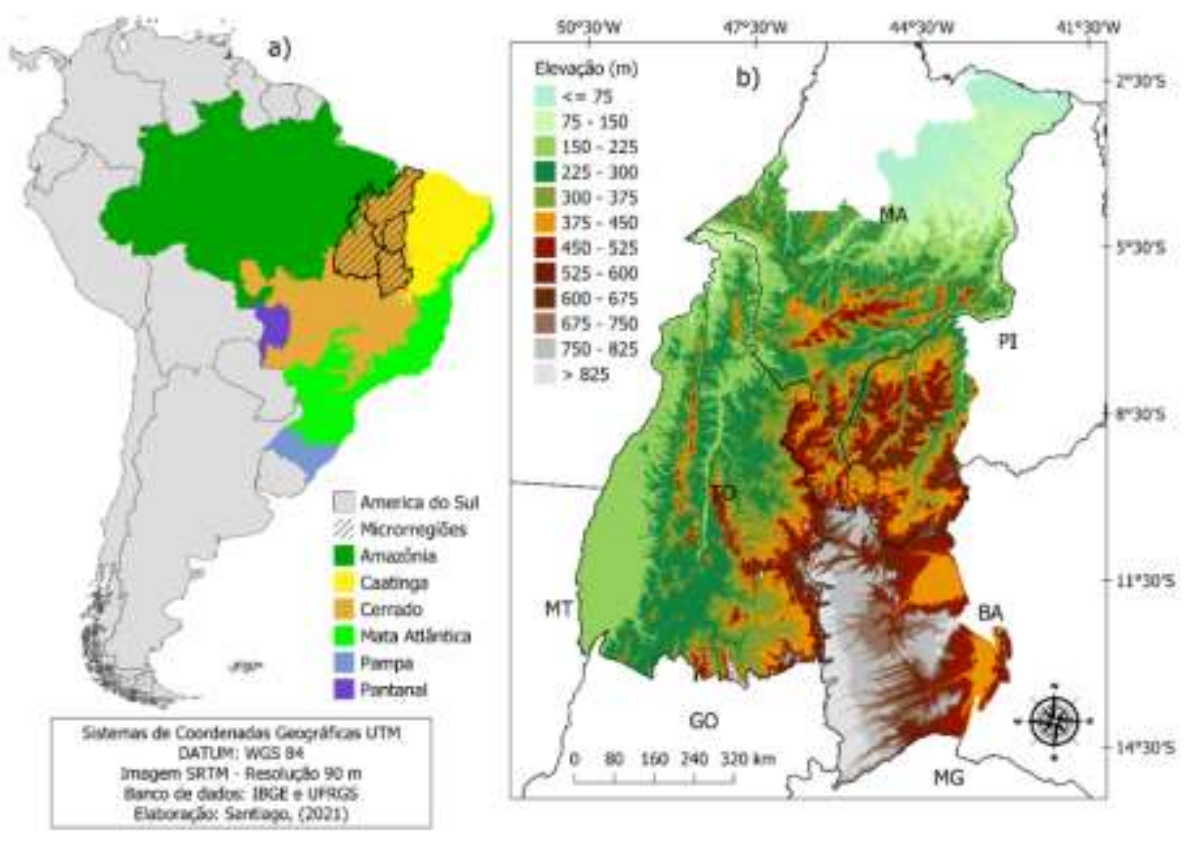

Fonte: Weber et al. (2004). Mapa elaborado pelos autores. 
Quanto ao clima da região é caracterizado por três zonas climáticas: tropical brasil central (53\%), tropical zona equatorial (44\%) e equatorial (3\%). Nestas, a extensão central do território, possui predominância do clima tropical semiúmido aproximadamente $78 \%$ do território, com temperaturas médias superiores a $18^{\circ} \mathrm{C}$ anualmente, com períodos de seca entre 4 e 5 meses. Já no limite leste é caracterizado por um clima semiárido, com baixa umidade e chuva (6 meses secos) e as temperaturas elevadas - (IBGE, 2002; Magalhães \& De Miranda, 2014).

O regime de chuvas da região é sazonal, com estações chuvosa e secas bem definidas, com chuvas superiores a 901 $\mathrm{mm}$ no período chuvoso, setembro/outubro até abril/maio do ano seguinte, e inferiores a $600 \mathrm{~mm}$ no período seco, meses de maio a setembro no restante do ano, onde praticamente não chove (Eiten, 1972; Lima, 2011; Nascimento e Novais, 2020).

\subsection{Dados}

Para a avaliação das interações acerca das alterações do uso e ocupação do solo, vegetação e a WUE, foram utilizadas as seguintes bases de dados: Elevação (SRTM), Precipitação (CHIRPS), Temperatura de superfície (MODIS), índice de vegetação (NDVI) e Uso e Ocupação do solo (Mapbiomas, 2021). Os produtos do satélite MODIS obtidos no USGS (USGS, 2021) são: MOD11A2 referentes a imagens da temperatura da superfície. Já os produtos MOD09A1 reúnem imagens da reflectância espectral da superfície, MOD17A2 a Produtividade Primária Bruta (GPP) e MOD16A2 a Evapotranspiração. Serão utilizadas as imagens da composição de oito dias para a criação de imagens mensais, e imagens anuais entre os anos de 2001 e 2019, com resolução espacial de 1km. Já o padrão espaço-temporal da precipitação será utilizado como auxílio dados do Climate Hazard Group InfraRed Precipitation with Station data (CHIRPS) - (Funk et al., 2015). Este possui uma resolução espacial de 5,6km x 5,6km, ao qual está disponível no seguinte endereço: <https://data.chc.ucsb.edu/products/CHIRPS-2.0/>. As realizações dos cálculos matemáticos serão executadas nos softwares ambiente R versão 3.6.3 (R Development Core Team, 2020) e Quantum GIS (QGIS) versão 3.4.6 (Qgis Core Team, 2019).

\subsection{Metodologia}

\subsubsection{Cálculo da Eficiência do Uso da Água (WUE)}

A estimativa de WUE pode ser obtida via métodos de volumes ou abordagens hidrológicas, baseadas na proporção da $\mathrm{H}_{2} \mathrm{O}$ utilizada pela planta em prol da sua produtividade (Koech \& Langat, 2018). A Figura 4, expõe os procedimentos para a obtenção da WUE. Assim sendo abordado como o rendimento de safra comercializável produzida por unidade de $\mathrm{H}_{2} \mathrm{O}$ usada como ET (Tilahun et al., 2011). Desta forma, será utilizada a razão entre GPP e ET (Equação 1) (Diaz et al., 2019; Xiangyang et al., 2019; Wang et al., 2020).

$$
\mathrm{WUE}=\frac{\mathrm{GPP}}{\mathrm{ET}}(1)
$$

\subsubsection{Cálculo do GPP via modelo de fotossíntese da vegetação (MFV)}

O modelo VPM (Vegetation Photosynthesis Model) desenvolvido por Xiao et al. (2004b), é um modelo de LUE Light Use Efficiency $\left(\mathscr{E}_{\mathrm{g}}\right)$ em gC.mol.PAR ${ }^{-1}$ (Liu et al., 2011) baseado em estimativas de satélites, descritas por parâmetros reguladores $\left(\mathrm{T}_{\text {escalar, }}, \mathrm{W}_{\text {escalar }}\right.$ e $\mathrm{P}_{\text {escalar }}$ ) e, assim permite o mapeamento diário da GPP (Chapin et al., 2011). Ele assume que as folhas e as copas das plantas são compostas de vegetação fotossinteticamente ativas (VFA), principalmente cloroplasto e vegetação não-fotossintética (VNF) principalmente senescência da folhagem, ramos e caules (Xiao et al., 2004b). Com base na partição conceitual de VFA e VNA dentro das copas, o GPP via MFV foi calculado a partir da equação 2:

$$
\mathrm{GPP}=\mathcal{\varepsilon} . \text { FPAR } . \text { PAR (2) }
$$


em que, FPAR é a fração de PAR ( $\mu$ mol. $\mathrm{m}^{2}$ ) absorvida pelo dossel da planta, o produto de FPAR e PAR é o PAR absorvido pelo dossel da planta (APAR), e $\mathscr{E}_{\mathrm{g}}$ é a eficiência de uso de luz (LUE) - (Vetrita et al., 2011).

O cálculo de FPAR depende de uma relação linear com EVI. Geralmente o coeficiente $\alpha$ é definido como 1, conforme descrito por Xiao et al. (2004). Onde:

$$
\operatorname{FPAR}=\alpha \cdot \operatorname{EVI}(3)
$$

O MFV adquire $\mathscr{E}$ máximo específico da literatura ou da relação entre a troca líquida do ecossistema (NEE) e incidente PAR; a temperatura e o estresse hídrico são escolhidos para regular $\mathscr{E}$ max. Assim, no MFV, $\mathscr{E}_{\mathrm{g}}$ é descrito como:

$$
\mathcal{E}=\mathcal{E}_{\text {máximo }} \cdot \mathrm{T}_{\text {escalar }} \cdot \mathrm{W}_{\text {escalar }} \cdot \mathrm{P}_{\text {escalar }}(4)
$$

em que, $\mathscr{E}_{\max }$ é o LUE máximo; e $\mathrm{T}_{\text {escalar }}, \mathrm{W}_{\text {escalar }}$ e $\mathrm{P}_{\text {escalar }}$ são os escalares de regulação baixa para a eficiência de temperatura, água e fenologia foliar no $\mathscr{E}_{\max }$, respectivamente (Yan et al., 2009; Wang et al.,2010; Madugundu et al., 2017).

Para estimar o $\mathrm{T}_{\text {escalar, }}$ usou-se a Ta é a temperatura média para cada um dos sete dias (semanal); $\mathrm{T}_{\min }, \mathrm{T}_{\max }$ e $\mathrm{T}_{\mathrm{opt}} \mathrm{são}$ os mínimos, temperatura máxima e ótima do ar para atividades fotossintéticas, respectivamente. Se $\mathrm{T}_{\mathrm{a}}$ for inferior a $\mathrm{T}_{\text {min }}, \mathrm{T}_{\mathrm{scaled}}$ é definido como 0 (Xiao et al, 2004c).

$$
\mathrm{T}_{\text {escalar }}=\frac{\left(\mathrm{T}_{\mathrm{a}}-\mathrm{T}_{\min }\right) \cdot\left(\mathrm{T}_{\mathrm{a}}-\mathrm{T}_{\max }\right)}{\left(\mathrm{T}_{\mathrm{a}}-\mathrm{T}_{\min }\right) \cdot\left(\mathrm{T}_{\mathrm{a}}-\mathrm{T}_{\max }\right)-\left(\mathrm{T}_{\mathrm{a}}-\mathrm{T}_{\mathrm{opt}}\right)^{2}}(5)
$$

O W $\mathrm{W}_{\text {escalar }}$ é o efeito da água para a fotossíntese da planta, assim se baseado no cálculo do LSWI, onde o LSWI max é o LSWI máximo dentro da estação de cultivo (Xiao et al., 2003).

$$
\mathrm{W}_{\text {escalar }}=\frac{1+\mathrm{LSWI}}{1+\mathrm{LSWI}_{\max }}(6)
$$

Durante a expansão total da folha, $\mathrm{P}_{\text {escalar }}=1$. Onde é o efeito da fenologia na fotossíntese do dossel (Xiao et al., 2004b).

$$
\mathrm{P}_{\text {escalar }}=\frac{(1+\mathrm{LSWI})}{2}(7)
$$

\section{Resultados e Discussão}

\subsection{Distribuição espacial da WUE}

A Figura da WUE (Figura 2), mostrou a distribuição espacial da WUE média entre os anos de 2001 a 2019 , sendo a WUE a razão entre a absorção de carbono e a perda de água da vegetação. A WUE varia entre 0 e 5 entre os anos de 2001 e 2019. A região onde ocorrem os maiores valores (>=2,33 $\left.\mathrm{gC}^{\mathrm{kgH}} \mathrm{kg}_{2}^{-1}\right)$ ocupam $11,93 \%\left(89.402,38 \mathrm{~km}^{2}\right)$ da área do MATOPIBA (Tabela 1), e encontram-se inseridos no oeste baiano (área sul) sobre regiões agrícolas, bem como na porção norte, a qual está relacionada a área de vegetação nativa amazônica. Sendo verificado em todos os anos de estudo (2001 a 2019). A classe predominante 1,67 - 2 gC. $\mathrm{kgH}_{2} \mathrm{O}^{-1}$ ocupa $50,47 \%\left(377.729,4 \mathrm{~km}^{2}\right)$ da área total do MATOPIBA e está relacionada a vegetação Savânica. Já os menores valores $\left(<=0,33 \mathrm{gC} \cdot \mathrm{kgH}_{2} \mathrm{O}^{-1}\right)$ ocupam 1,08\% $\left(8.093,89 \mathrm{~km}{ }^{2}\right)$ da área, e estão dispostos em área de rios, lagos e áreas de solo sem vegetação, como na porção noroeste do MATOPIBA, situados na região litorânea do Maranhão (Nordeste). 
Tabela 1 - Valores das classes referente à WUE médio de 2001 a 2019 juntamente com as porcentagens $(\%)$ e as áreas $\left(\mathrm{km}^{2}\right)$ ocupadas.

\begin{tabular}{|c|c|c|c|c|c|}
\hline Classes WUE & $\mathrm{km}^{2}$ & $\%$ & Classes WUE & $\mathrm{km}^{2}$ & $\%$ \\
\hline 0 & 4684,96 & 0,63 & $1,33-1,67$ & 58550,55 & 7,81 \\
\hline $0-0,333$ & 3408,93 & 0,45 & $1,67-2$ & 377729,4 & 50,40 \\
\hline $0,333-0,667$ & 2046,37 & 0,27 & $2-2,33$ & 207191,7 & 27,65 \\
\hline $0,667-1$ & 1371,9 & 0,18 & $2,33-2,67$ & 85012,68 & 11,34 \\
\hline $1-1,33$ & 5005,87 & 0,67 & $>=2,67$ & 4389,7 & 0,59 \\
\hline
\end{tabular}

Fonte: Autores (2021).

O padrão de mínimos e máximos da WUE está relacionado a diferentes vegetações que possuem diferentes absorções de Carbono e água, motivados pela sua estrutura fisiológica (Tong et al., 2009; Taub, 2010). Em áreas agrícolas por possuírem um manejo mais bem definido, obtêm-se os maiores valores da WUE quando comparado por exemplo com áreas de vegetações nativas. Já áreas de rios, lagos e sem vegetação (solo nu), não promovem fotossíntese, assim encontra-se os menores e próximos de zero.

Figura 2 - Distribuição espacial média da WUE de 2001 a 2019.

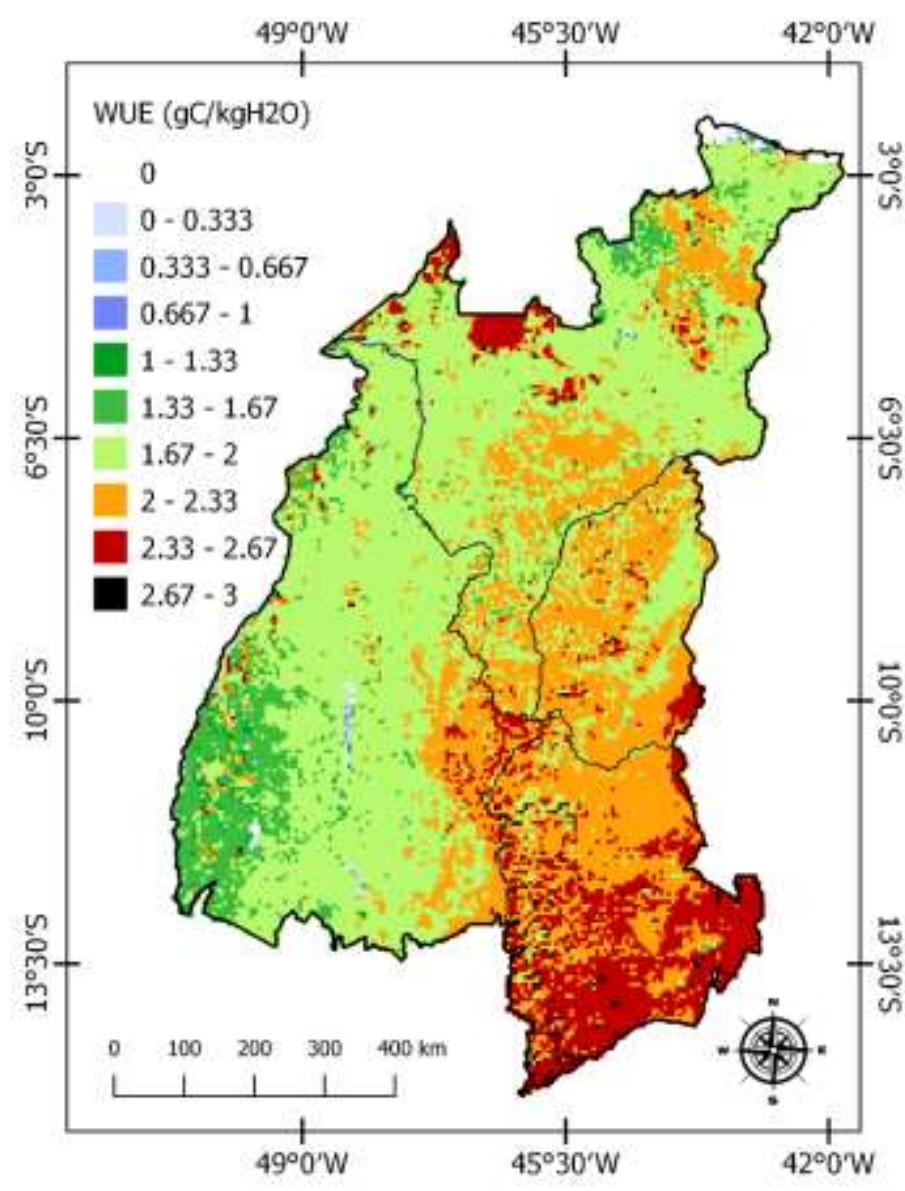

Fonte: Autores (2021).

Em escala anual (Figura 3), os anos de 2016 e 2017 mostraram os maiores valores médios (entre $2-2,20$ gC.kgH $\mathrm{g}^{-1}$ ) da WUE na área do oeste baiano e a porção nordeste do MATOPIBA. Nessa área os maiores valores são encontrados em 
virtude do plantio agrícola, com destaque para a soja, cultura em expansão no MATOPIBA. Ao longo de 19 anos houve um aumento de 2,47\% (34.958 $\left.\mathrm{km}^{2}\right)$, disposta na Figura 4.

Figura 3 - Valores máximos e médios da WUE entre os anos de 2001 e 2019.

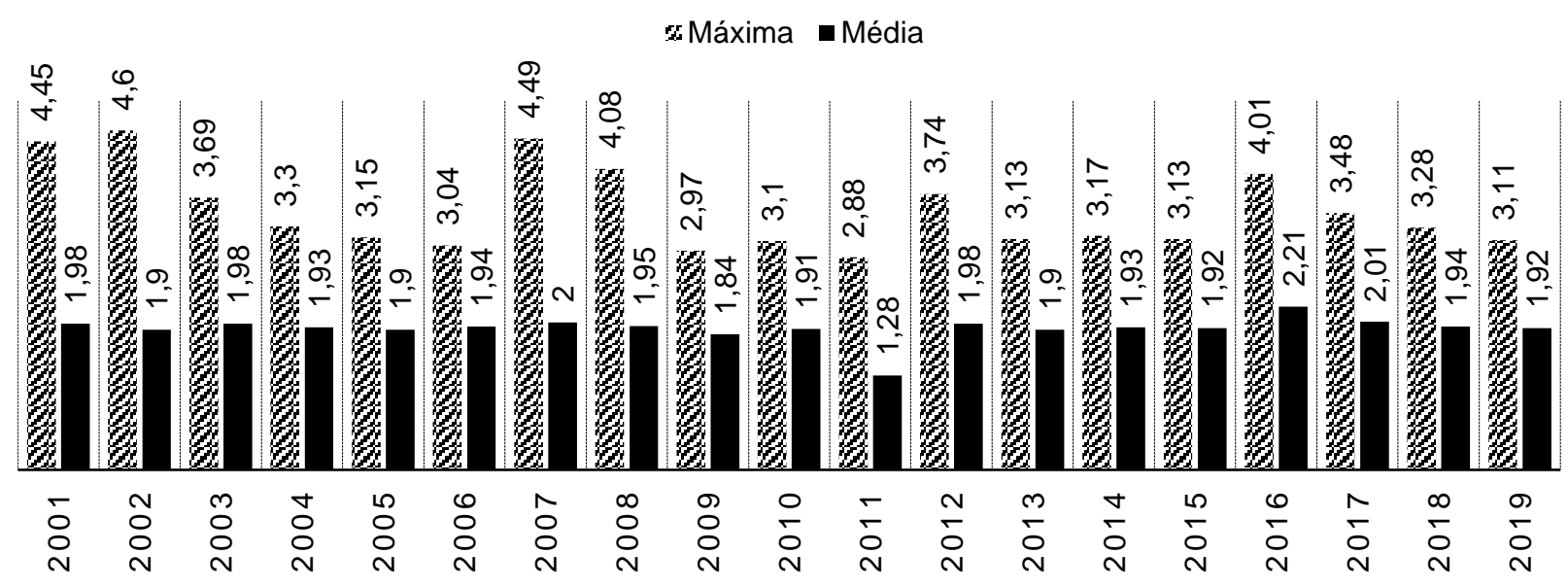

Fonte: Autores (2021).

\subsection{Variabilidade espaço-temporal do uso e ocupação do solo.}

Os resultados referentes a variabilidade da WUE, estão relacionados a variabilidade do uso e ocupação do solo na região do MATOPIBA. Onde foi observado utilizando dados de uso e ocupação do solo do MAPBIOMAS entre os anos de 2001 e 2019 (Figura 4) e detalhada na Tabela 2. A variação espacial da WUE, sofre o efeito da mudança do uso e ocupação do solo para fins agrícolas. A Figura 4a exibe a cobertura do solo referente ao ano de 2001, ao qual aponta o predomínio da classe de floresta natural (Nordeste do Maranhão), com 505,207 km² (35,65\%), já a agropecuária 7,59\% (107,643 km²), a agricultura $0,52 \%\left(7,393 \mathrm{~km}^{2}\right)$ e plantio de soja $0,53 \%\left(7581 \mathrm{~km}^{2}\right)$.

Ao longo dos 19 anos (Figura 4a e 4b), houve diminuição na área $\left(\mathrm{em}^{\mathrm{k}} \mathrm{km}^{2}\right)$ da classe floresta natural de aproximadamente 4,92\%, devido ao aumento de áreas com produções agrícolas. Em contrapartida, o crescimento dos outros tipos de ocupação ficou claro quando observados ao longo dos 19 anos (Tabela 2 e Figura 4). Entre o período de 2001 e 2019, houve aumento de 2,54\% (35875 $\left.\mathrm{km}^{2}\right)$ na área ocupada por agropecuária, de 0,56\% (7976 km²) na agricultura, e 2,47\% (34958 $\left.\mathrm{km}^{2}\right)$ no Plantio de Soja.

Algumas projeções indicam que entre as safras 2013/2014 e 2023/2024, a produção de grão no MATOPIBA terá uma alta de 21,4\%, saltando de 18,6 milhões de toneladas verificados na safra 2013/2014 para 22,6 milhões de toneladas no ciclo 2023/2024 (PA, 2017). 
Figura 4 - Classificação do uso e ocupação do solo para os anos de 2001 e 2019.

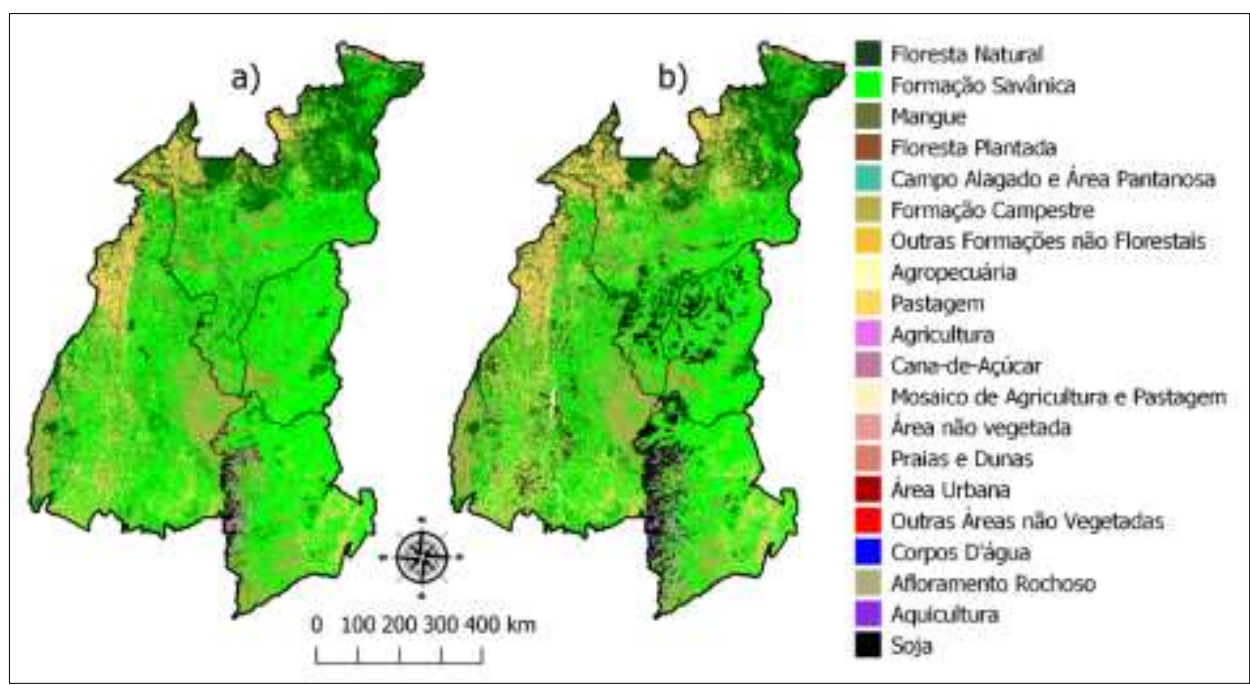

Fonte: Dados MAPBIOMAS. Elaboração Autores (2021).

Tabela 2 - Valores das classes referente ao tipo de ocupação do solo, juntamente com as porcentagens $(\%)$ e as áreas $\left(\mathrm{km}^{2}\right)$ ocupadas.

\begin{tabular}{c|c|c|c|c}
\hline & \multicolumn{2}{|c|}{2001} & \multicolumn{2}{c}{2019} \\
\hline Tipos de Ocupação & $\mathrm{km}^{2}$ & $\%$ & $\mathrm{~km}^{2}$ & $\%$ \\
\hline Floresta natural & 505207 & 35,65 & 435581 & 30,73 \\
Agropecuária & 107643 & 7,59 & 143518 & 10,13 \\
Agricultura & 7393 & 0,52 & 15369 & 1,08 \\
Soja & 7581 & 0,53 & 42539 & 3,00 \\
Outros & 789477 & 55,70 & 780294 & 55,05 \\
\hline
\end{tabular}

Fonte: Autores (2021).

\subsection{Distribuição espaço-temporal das anomalias da WUE.}

A variação da WUE também pode ser alterada em virtude dos aspectos relacionados a variabilidade da precipitação. Já que a absorção de carbono e a perda de água possui uma forte relação, implicando assim, que uma perturbação nas componentes da WUE (GPP e ET), impacta diretamente na WUE. Desta forma, anos mais secos e chuvosos alteram a disponibilidade pluviométrica e de umidade no solo, impactando significativamente no ciclo do carbono e água. A Figura 5 mostra a distribuição espacial das anomalias da WUE referentes a anos de El Niño (2003, 2007 e 2016) e La Niña (2005, 2010 e 2012).

Os El Niños causam períodos de estiagens no nordeste brasileiro, ao qual causam redução da disponibilidade hídrica pluviométrica e altas temperaturas locais (De Melo, 1999), enquanto os La Niñas promovem a presença de grandes volumes pluviométricos (Freire et al., 2011; Marengo et al., 2016; Correia Filho et al., 2019), onde essas alterações impactam diretamente na ET e GPP, posteriormente na WUE. Durante os anos secos (El Niño) foram observados valores de anomalias positivas superiores às negativas (Tabela 3), onde o ano de 2003 (+54\%), $2007(+56 \%)$ e 2016 (+97\%), indica em anos mais secos a vegetação produz os maiores valores da WUE em períodos com precipitações, corroborando com alguns estudos que mencionam este fato (Ponce-Campos et al., 2013; LIU et al., 2015).

Em contrapartida, em anos chuvosos (La Niña) chuvas acima da média, principalmente no nordeste brasileiro, ao qual resultam anomalias negativas da WUE (-69\%) 2005 e (-62\%) 2010. Este resultado ocorre devido ao excesso de chuvas que 
contribui para a escassez de oxigênio no solo, reduzindo a decomposição da matéria orgânica e, subsequentemente, o fornecimento de nutrientes (schuur \& Matson, 2001). O que interfere nos processos do crescimento da planta, como entrada de radiação, lixiviação de nutrientes ou oxigênio do solo (Schuur et al., 2001). Além da alta presença de nuvens associadas, diminuir a radiação solar, prejudicando os processos fotossintéticos e sua alta produtividade. Já o ano de 2012 foi um ano atípico de La Niña apresentando uma forte irregularidade nos regimes pluviométricos (Marengo et al., 2016), corroborando com os percentuais positivos (59\%). Carrão et al. (2016) identificaram uma forte diminuição da umidade do solo no La Niña de 2012, sendo comparado a um El Niño ocorrido em 1997-1998.

Na figura 6 disposta em escala mensal, pode-se observar esta relação entre a variação da WUE e a precipitação, ao qual os meses com valores inferiores a $150 \mathrm{~mm}$ precipitações (julho, agosto e setembro), obtiveram os maiores valores da WUE médio (>= $\left.6 \mathrm{gC} \cdot \mathrm{kgH}_{2} \mathrm{O}^{-1}\right)$, corroborando com as respostas da vegetação aos períodos de baixa ou zero pluviosidade e vice versa e seu melhor desempenho. Os meses com chuvas superiores a $300 \mathrm{~mm}$, a WUE apresentou valores menores que 4 $\mathrm{gC} \cdot \mathrm{kgH}_{2} \mathrm{O}^{-1}$. Já que o período chuvoso é de setembro/outubro até abril/maio do ano seguinte, e o período seco, nos meses de maio a setembro, onde se tem baixas pluviosidades ou nenhuma (Nascimento \& Novais, 2020).

Tabela 3 - Percentuais positivos e negativos das anomalias.

\begin{tabular}{l|l|l|l|l|l|l}
\hline & \multicolumn{3}{c|}{ Anos Secos } & \multicolumn{3}{c}{ Anos Chuvoso } \\
\hline & 2003 & 2007 & 2016 & 2005 & 2010 & 2012 \\
\hline Negativos & $46 \%$ & $44 \%$ & $3 \%$ & $69 \%$ & $62 \%$ & $41 \%$ \\
Positivos & $54 \%$ & $56 \%$ & $97 \%$ & $31 \%$ & $38 \%$ & $59 \%$ \\
\hline
\end{tabular}

Fonte: Autores (2021).

Figura 5 - Distribuição espacial das anomalias da WUE durante os períodos secos (2003, 2007 e 2016) e chuvoso (2005, 2010 e 2012) na Região do MATOPIBA.

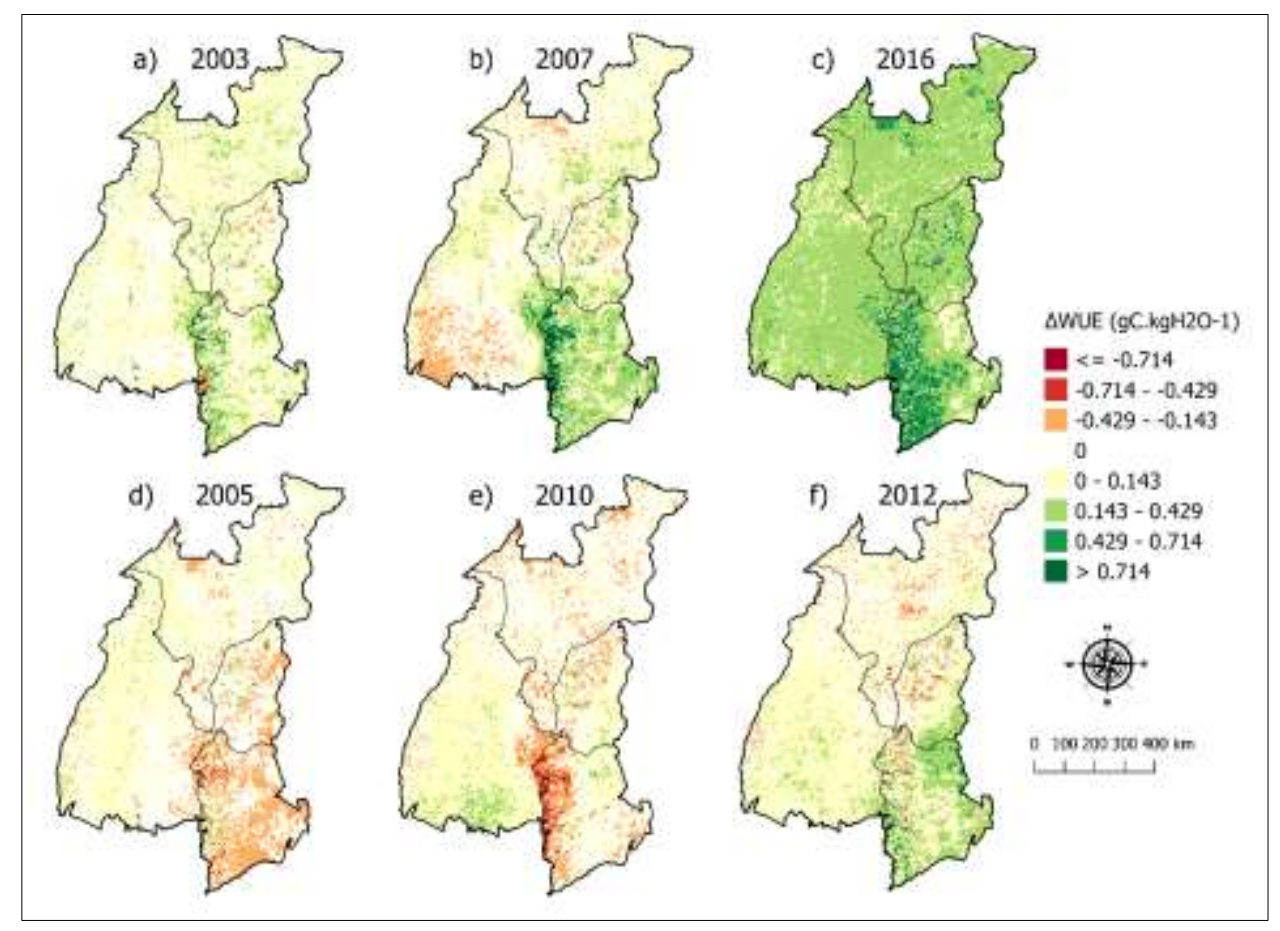

Fonte: Autores (2021). 
Figura 6 - Média mensal da precipitação e QUE, durante o período entre 2001 e 2019.

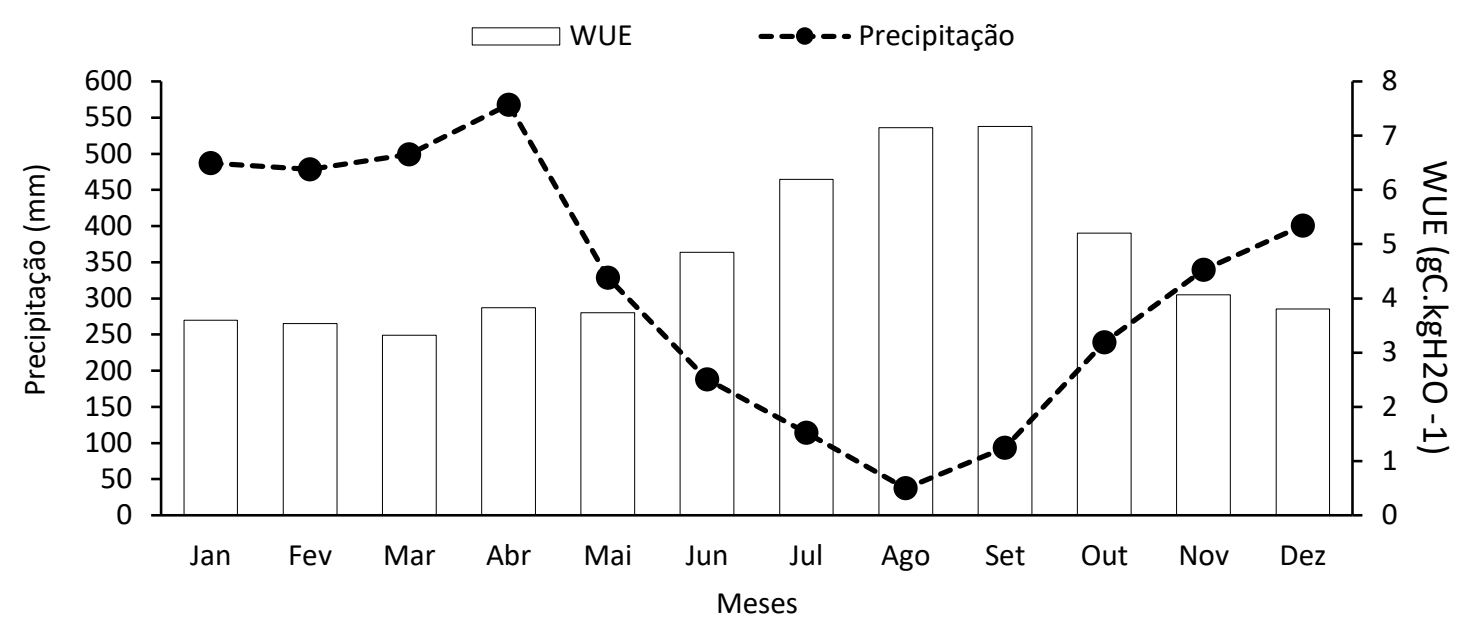

Fonte: Autores (2021).

De uma forma geral, os maiores valores da WUE foram observados na região do oeste baiano (sul do MATOPIBA), favorecidos pela elevação e o manejo agrícola. As regiões elevadas favorecem a WUE, devido ao fato de impactar na evapotranspiração de forma negativa, ou seja, a evapotranspiração diminui com a altura. Dessa forma se obtém os maiores valores de GPP. Consequentemente, a maior absorção de carbono (GPP), e maiores valores da WUE, também associados as alterações no uso e ocupação do solo. A variação negativa da GPP e ET com a elevação, pode ser explicada, devido a relação negativa da temperatura do ar e a precipitação (Bourque \& Mir, 2012). À medida que ocorrem as substituições da vegetação natural por áreas agrícolas, têm-se a implementação de irrigação. A irrigação favorece o acoplamento de carbono pela vegetação. A simples melhora de $1 \%$ na eficiência do uso da água de irrigação, nos países em desenvolvimento de clima semiárido ou árido, significaria uma economia de 200 mil litros de água, por agricultor, por hectare/ano (Coelho, Coelho Filho \& Oliveira, 2005) aumentando a WUE de áreas agrícolas.

\section{Considerações Finais}

A variação espacial da WUE ao longo de 19 anos, mostrou uma variação heterogenia causadas pelas modificações do uso e ocupação. Principalmente quando relacionados a expansão agrícola, nesse caso, áreas do plantio de soja foram o destaque. As áreas onde ocorreram a substituição da vegetação natural por áreas agrícolas, mostrou um aumento da WUE. Indicando que áreas agrícolas associadas ao bom manejo tendem a ter melhores respostas da eficiência do uso da água pelas culturas. A área de formação florestal diminuiu ao longo dos anos de 2001 a 2019 (4,92\%), isso relacionado a expansão da agricultura. Já áreas agrícolas tiveram grande avanço, onde plantio de soja se destacou na ocupação de grande parte do MATOPIBA, principalmente nas áreas do oeste baiano e na divisa Maranhão-Piauí. Respostas acerca de como a vegetação é modificada quando ocorrem eventos de secas ou chuvas e a influência na WUE, mostraram que áreas agrícolas são mais sensíveis, tendo maiores valores da WUE em períodos de baixa pluviosidade, e menos em áreas de vegetação nativas, e impactados por períodos de alta pluviosidade.

O presente estudo mostra que avaliando a eficiência do uso da água (WUE), têm-se ótimas respostas sobre áreas agrícolas, o que serve de auxílio para um melhor entendimento sobre como ocorre a relação de absorção de carbono e a perda por evaporação das culturas. Servindo assim, de base para outros estudos relacionados a culturas agrícolas, não descartando a utilização em áreas florestais. 


\section{Referências}

Bastiaanssen, W. G. \& Ali, S. (2003). A New Crop Yield Forecasting Model Based On Satellite Measurements Applied Across The Indus Basin, Pakistan. Agriculture, Ecosystems \& Environment, 94, 321-340.

Bourque, C. P. A., \& Mir, M. A. (2012). Seasonal snow cover in the Qilian Mountains of Northwest China: Its dependence on oasis seasonal evolution and lowland production of water vapour. Journal of Hydrology, 454, 141-151.

Carrão, H., Russo, S., Sepulcre-Canto, G. \& Barbosa, P. (2016). An mpirical standardized soil moisture index for agricultural drought assessment from emotely sensed data. International Journal of Applied Earth Observation and Geoinformation, 48, 74-84.

Chapin III, F. S., Matson, P. A., Mooney, H. A. (2002). Principles Of Terrestrial Ecosystem Ecology. Berlin, Germany: Springer-Verlag, 2002.436 P.

Coelho, E. F., Coelho Filho, M., A., \& De Oliveira, S. L. (2005). "Agricultura Irrigada: Eficiência De Irrigação E De Uso De Água. " Bahia Agrícola 7. 1: 5760.

Colussi, J. (2017). MATOPIBA: Mudanças No Uso Da Terra Na Nova Fronteira.

CONAB - Companhia Nacional de Abastecimento. (2019). Acompanhamento da Safra Brasileira de Grãos, Safra 2018/19 - Oitavo Levantamento, 1-135, https://www. conab. gov. br/info-agro/safras/graos/boletim-da-safra-de-graos.

Correia Filho, W. L. F., Barros Santiago, D., Oliveira-Júnior, J. F., \& Silva Junior, C. A. (2019). Impact Of Urban Decadal Advance On Land Use And Land Cover And Surface Temperature In The City Of Maceió, Brazil. Land Use Policy, 87, 104026. Https://Doi. Org/10. 1016/J. Landusepol. 2019. 104026.

Correia Filho, W. L. F., De Oliveira-Júnior, J. F., De Barros Santiago, D., De Bodas Terassi, P. M., Teodoro, P. E., De Gois, G., \& Dos Santos, P. J. (2019). Rainfall variability in the Brazilian northeast biomes and their interactions with meteorological systems and ENSO via CHELSA product. Big Earth Data, 3(4), 315-337. Https://Doi. Org/10. 1080/20964471. 2019. 1692298.

Dalastra, G. M., De Moraes Echer, M., Guimarães, V. F., Brito, T. S., \& Inagaki, A. M. (2020). Trocas gasosas e produtividade de tomateiro com diferentes hastes por planta. Iheringia. Série Botânica. 75.

De Melo, J. C. (1999). O fenômeno El Niño e as secas no Nordeste do Brasil. Raízes: Revista de Ciências Sociais e Econômicas, (20), 13-21.

Diaz, M. B., Roberti, D. R., Carneiro, J. V., Souza, V. de A., \& de Moraes, O. L. L. (2019). Dynamics of the superficial fluxes over a flooded rice paddy in southern Brazil. Agricultural and Forest Meteorology, 276-277, 107650. 10. 1016/j. agrformet. 2019. 107650

Eiten. (1972). The Cerrado Vegetation Of Brazil. York, New Garden, Botanical, 38, 201-341.

Freire, J. L. M., Lima, J. R. A., \& Cavalcanti, E. P. (2011). Análise de aspectos meteorológicos sobre o Nordeste do Brasil em anos de El Niño e La Niña. Revista Brasileira de Geografia Física, 3, 429-444.

Funk, C., Peterson, P., Landsfeld, M., \&Michaelsen, J. (2015). The Climate Hazards Infrared Precipitation With Record For Monitoring Extremes. Scientific Data, 2, 10-66.

IBGE. (2002). Mapa De Clima Do Brasil. Http://Www. Visualizador. Inde. Gov. Br/

Kijne, J. W., Barker, R. \& Molden, D. J. (2003). Water productivity in agriculture: limits and opportunities for improvement. Cabi.

Koech, R., Langat, P. (2018). Review Improving Irrigation Water Use Efficiency: A Review Of Advances, Challenges, And Opportunities In The Australian.

Lima, J. E. F. W. (2011). "Situação E Perspectivas Sobre As Águas Do Cerrado. " Ciência E Cultura 63. 3: 27-29.

Liu, J., Sun O. J., Jin, H., Zhou, Z. \& Han, X. (2011). Application Of Two Remote Sensing GPP Algorithms At A Semiarid Grassland Site Of North China. Journal Of Plant Ecology. 4, 302-312.

Liu, Y., Xiao, J., Ju, W., Zhou, Y., Wang, S., \& Wu, X. (2015). Water use efficiency of China's terrestrial ecosystems and responses to drought. Scientific reports, 5(1), 1-12.

Madugundu R, Al-Gaadi K. A., Tola E. K., Kayad A. G., Jha C. S. (2017). Estimation Of Gross Primary Production Of Irrigated Maize Using Landsat-8 Imagery And Eddy Covariance Data. Saudi J Biol Sci 24:410-20.

Magalhães, L. A., De Miranda, E. E. (2014). MATOPIBA: Quadro Natural. Embrapa Territorial-Outras Publicações Técnicas (INFOTECA-E).

Marengo, J. A., Cunha, A. P., \& Alves, L. M. (2016). A seca de 2012-15 no semiárido do Nordeste do Brasil no contexto histórico. Climanálise, 3(1), 1-6.

Mbava, N., Mutema, M., Zengeni, R., Shimelis, H., \& Chaplot, V. (2020). Factors affecting crop water use efficiency: A worldwide meta-analysis. Agricultural Water Management, 228, 105878. 10. 1016/j. agwat. 2019. 105878

Miranda, E. E., Magalhães, L. A. \& Carvalho, C. A. De. (2014). Proposta De Delimitação Territorial Do MATOPIBA. Embrapa GITE. Https://Www. Embrapa. Br/Gite/Publicacoes/>

Miranda, E. E. (2015). Matopiba Delimitação, Caracterização, Desafios E Oportunidades Para O Desenvolvimento. Embrapa GITE. Https://Www. Embrapa. Br/Gite/Projetos/MATOPIBA/MATOPIBA. Html

Morison, J. I., Baker, N., Mullineaux, P., \& Davies, W. (2008). Melhorar O Uso Da Água Na Produção Agrícola. Philosophical Transactions Of The Royal Society B: Biological Sciences, 363 (1491), 639-658. 10. 1098 / Rstb. 2007. 2175 
Nascimento, D., Novais, G. (2020). Clima do Cerrado: dinâmica atmosférica e características, variabilidades e tipologias climáticas. Élisée - Revista De Geografia Da UEG, 9(2), e922021. https://www.revista.ueg.br/index.php/elisee/article/view/10854

Nicodemo, M., De Moraes, L. F. D., De Oliveira, R. E., \& De Queiroga, J. L. (2021). Tecnologias agroecológicas apropriadas para a transição agroecológica na agricultura familiar. Embrapa Pecuária Sudeste-Documentos (INFOTECA-E).

Ponce-Campos, G. E., Moran, M. S., Huete, A., Zhang, Y., Bresloff, C., Huxman, T. E., \& Starks, P. J. (2013). Ecosystem resilience despite large-scale altered hydroclimatic conditions. Nature, 494(7437), 349-352.

PA (Projeções do Agronegócio). (2017). Brasil 2016/17 a 2026/27. Projeções de longo prazo. MAPA (8a ed.), 125 p.

Projeto MapBiomas. (2021) Coleção 5 da Série Anual de Mapas de Cobertura e Uso de Solo do Brasil. Disponível em: https://mapbiomas. org/colecoesmapbiomas. Acesso em 10 jan. 2021.

QGIS - Quantum Geographic Information System. (2019). Quantum GIS Geographic Information System. V. 3. 4. 6. Open Source Geospatial Foundation Project.

R Development Core Team, (2020). R: A Language And Environment For Statistical Computing. R Foundation For Statistical Computing, Vienna, Austria, Http://Www. R-Project. Org, ISBN 3-900051-07-0.

Sá, H., Morais, L. \& Campos, C. (2015). Que Desenvolvimento É Esse? Análise Da Expansão Do Agronegócio Da Soja Na Área Do MATOPIBA A Partir De Uma Perspectiva Furtadiana. In: Anais Do XXI Congresso Brasileiro De Economia.

Schuur, E. A. \& Matson, P. A. (2001). Net primary productivity and nutrient cycling across a mesic to wet precipitation gradient in Hawaiian montane forest. Oecologia, v. 128, 431-442.

Schuur, E. A., Chadwick, O. A. \& Matson, P. A. (2001). Carbon cycling and soil carbon storage in mesic to wet Hawaiian montane forests. Ecology, 82, 31823196.

Tang X., Li H., Desai A. R., \& Ammann C. (2015a). How Is Water-Use Efficiency Of Terrestrial Ecosystems Distributed And Changing On Earth? Science Reports 4:7483.

Tang, X., Ding, Z., Li, H., Li, X., Luo, J., Xie, J., \& Chen, D. (2015b). Characterizing Ecosystem Water-Use Efficiency Of Croplands With Eddy Covariance Measurements And MODIS Products. Ecological Engineering, 85, 212-217. 10. 1016/J. Ecoleng. 2015. 09. 078.

Taub, D. (2010). Effects of rising atmospheric concentrations of carbon dioxide on plants. Nature Education Knowledge, v. 1.

Tilahun, H., Teklu, E., Michael, M., Fitsum, H., \& Awulachew, S. B., (2011). Comparative Performance Of Irrigated And Rainfed Agriculture In Ethiopia. World Appl. Science. J. 14 (2), 235-244.

Tong, X. -J., Li, J., Yu, Q., \& Qin, Z. (2009). Ecosystem water use efficiency in an irrigated cropland in the North China Plain. Journal of Hydrology, 374(34), 329-337. 10. 1016/j. jhydrol. 2009. 06. 030

USGS. United States Geological Survey. Earth Explorer. (2021). https://earthexplorer. usgs. gov〉.

Vetrita, Y., Chaoyang, W., Zheng, N. \& Hirano, T. (2011). Evaluation Of Light Use Efficiency Model Using Modis In Tropical Peat Swamp Forest, Indonesia. In: Second Cresos International Symposium On South East Asia Environmental Problems And Satellite. Remote Sensing, Indonesia, 127-134.

Wang Z., Xiao X., \& Yan X. (2010). Modeling Gross Primary Production Of Maize Cropland And Degraded Grassland In Northeastern China. Agricultura Forest Meteorology 150:1160-7.

Wang, Y., Ma, Y., Li, H., \& Yuan, L. (2020). Carbon and water fluxes and their coupling in an alpine meadow ecosystem on the northeastern Tibetan Plateau. Theoretical and Applied Climatology, 142(1), 1-18.

Weber, E., Hasenack, H. \& Ferreira, C. J. S. (2004). Adaptação do modelo digital de elevação do SRTM para o sistema de referência oficial brasileiro e recorte por unidade da federação. Porto Alegre. UFRGS Centro de Ecologia. https://www.ufrgs. br/labgeo/index.php/dados-espaciais/260-modelos-digitaisde-elevacao-do-srtm-no-formato-geotiff $>$.

Xiangyang, S., Genxu, W., Mei, H., Ruiying, C., Zhaoyong, H., Chunlin, S., \& Juying, S. (2019). The asynchronous response of carbon gain and water loss generate spatio-temporal pattern of WUE along elevation gradient in southwest China. Journal of Hydrology, 124389. 10. 1016/j. jhydrol. 2019. 124389

Xiao, G., Zheng, F., Qiu, Z., \& Yao, Y. (2013). Impact of climate change on water use efficiency by wheat, potato and corn in semiarid areas of China. Agriculture, Ecosystems \& Environment, 181, 108-114. 10. 1016/j. agee. 2013. 09. 019

Xiao, X., Braswell, B., Zhang, Q., Boles, S., Frolking, S., \& Moore, B. (2003) Sensitivity Of Vegetation Indices To Atmospheric Aerosols: Continental- Scale Observations In Northern Asia. Remote Sensing Of Environment. 84, 385-392.

Xiao, X., Zhang, Q., Braswell, B., Urbanski, S., Boles, S., Wofsy, S., Moore Iii, B. \& Ojima, D. (2004c). Modeling Gross Primary Production Of Temperate Deciduous Broadleaf Forest Using Satellite Images And Climate Data. Remote Sensing Of Environment. 91, 256-270.

Xiao, X., Zhang, Q., Hollinger, D., Aber, J., \& Moore Iii, B. (2004b). Modeling Seasonal Dynamics Of Gross Primary Production Of An Evergreen Needleleaf Forest Using MODIS Images And Climate Data. Ecological Applications. 15, 954-969.

Yamori, W. (2020). Photosynthesis and respiration. Plant Factory, 197-206. 10. 1016/b978-0-12-816691-8. 00012-1

Yan H, Fu Y, Xiao X, Huang HQ, He H, \& Ediger L. (2009). Modeling Gross Primary Productivity For Winter Wheat-Maize Double Cropping System Using MODIS Time Series And CO2 Eddy Flux Tower Data. Agric Ecosyst Environ 129:391-400.

Zwart, S. J., \& Bastiaanssen, W. G. M. (2004). Review of measured crop water productivity values for irrigated wheat, rice, cotton and maize. Agricultural Water Management, 69(2), 115-133. 10. 1016/j. agwat. 2004. 04. 007 